\title{
Der Clamp Rod Internal Fixator zur Behandlung von Pseudarthrosen beim Kleintier
}

\author{
Klaus Zahn, Ulrike Matis
}

\section{Zusammenfassung}

Pseudarthrosen nach Osteosynthese langer Röhrenknochen treten bei Hund und Katze mit einer Häufigkeit von $1-2 \%$ auf und beruhen meistens auf operationstechnischen Fehlern. Die Behandlungsstrategie folgt dem Debridement des Frakturspaltes und der Einbringung autogener Spongiosa in Verbindung mit einer stabilen Fixation der Fraktur. Mit dem neuen, speziell für die Tiermedizin entwickelten Clamp Rod Internal Fixator (CRIF) konnte bei 11 Hunden und 3 Katzen mit Pseudarthrose - darunter 8 infi- ziert und 3 atrophisch - im Mittel nach 11 Wochen eine störungsfreie Konsolidierung dokumentiert werden. Das CRIF-System entspricht einem Fixateur interne und besteht aus Klammern, die auf einen Rundstab aufgeschoben und mit Schrauben am Knochen fixiert werden. Es zeichnet sich durch hohe Vielseitigkeit und geringen Implantat-Knochenkontakt aus und verfolgt damit im Sinne moderner Osteosynthesekonzepte die Philosophie eines möglichst ungestörten periostalen Blutflusses bei zugleich stabiler Fixation.

\section{Einleitung}

Hunde und Katzen mit Frakturen der langen Röhrenknochen zählen zum regelmäßigen Patientengut einer städtischen Kleintierpraxis oder -klinik. Die Tiere werden meist polytraumatisiert nach Autounfällen oder nach Sturz aus größerer Höhe vorgestellt und überwiegend operativ therapiert, um eine rasche Wiederherstellung der Gliedmaßenfunktion zu erreichen. $\mathrm{Zu}$ den am häufigsten angewandten Verfahren zählen die Plattenosteosynthese, die Marknagelung sowie die perkutane Transfixation. Verriegelungsnägel gehören aufgrund ihrer limitierten Einsetzbarkeit beim Tier nicht zur Standardausstattung einer chirurgischen Praxis $[2,4,10,16]$.

Die Komplikationsrate nach Osteosynthese langer Röhrenknochen beim Kleintier liegt einer Studie mit 1005 Patienten zufolge bei etwa $12-16 \%$ [10]. Während die Osteomyelitis beim Hund mit 38\%

OP-JOURNAL 2004; 20: 128-132

(c) Georg Thieme Verlag KG Stuttgart · New York am häufigsten festgestellt wurde, überwogen bei der Katze Implantatlockerungen (45\%). Die Pseudarthrose stellte unter den Komplikationen bei beiden Spezies einen Anteil von 10-13\% und betraf überwiegend Femur und Tibia. Als Hauptursache für das Entstehen einer Pseudarthrose gilt beim Kleintier ungenügende Stabilität aufgrund operationstechnischer Fehler [1,13]. Meist finden sich hypertrophe Pseudarthrosen, die nicht selten infiziert sind. Oligotrophe und atrophe Formen kommen seltener vor, beispielsweise als Defektpseudarthrose nach Knochenverlust und nach offenen Frakturen [11,15,16,19].

Während hierzulande die Zahl der Osteosynthesekomplikationen beim Kleintierpatienten über die Jahre hinweg stetig abnahm, werden heute vermehrt Hunde mit Frakturheilungsstörungen nach Osteosynthese vorgestellt, die von Urlaubsreisenden aus südlichen Ländern importiert wurden. Im Allgemeinen erfolgt bei diesen Patienten im Herkunftsland mangels finanzieller Möglichkeiten und chirurgischer Erfahrung keine Revision, wodurch es schließlich zur Pseudarthrose kommt.
Im Rahmen einer klinischen Prüfung des neuen Clamp Rod Internal Fixator (CRIF) [23] wurden elf Hunde und drei Katzen mit nicht heilender Fraktur nach Osteosynthese der langen Röhrenknochen in ihrem Heilungsverlauf verfolgt, der den CRIF als ein speziell für diese Situationen geeignetes, hier darstellungswürdiges Implantat erscheinen lässt.

\section{Der Clamp Rod Internal Fixator}

Der Clamp Rod Internal Fixator (CRIF) wurde 1998 vom AO-Entwicklungsinstitut in Davos für die interne extrakortikale Osteosynthese beim Tier konzipiert und wird am besten mit „Fixateur interne“ beschrieben. Bislang gab es nur Prototypen des Implantats, die einigen Universitätstierkliniken zur Verfügung gestellt wurden.

Das System besteht aus Klammern, die auf einen Rundstab aufgeschoben und entlang des Stabes beliebig angeordnet werden können (Abb.1).

Die Klammern werden mit Standardschrauben beim Festziehen zusammengedrückt und so der Stab in der Klammer und am Knochen fixiert (Abb.2).

Das CRIF-System stand zur Testung für Schraubengrößen von 2,0, 2,7, 3,5 und $4,5 / 5,5 \mathrm{~mm}$ und einem Stabkaliber von 2, 3, 5 und $8 \mathrm{~mm}$ zur Verfügung [22]. Im Verlauf der Prüfung wurden zusätzlich Endklammern sowie spezielle Instru-

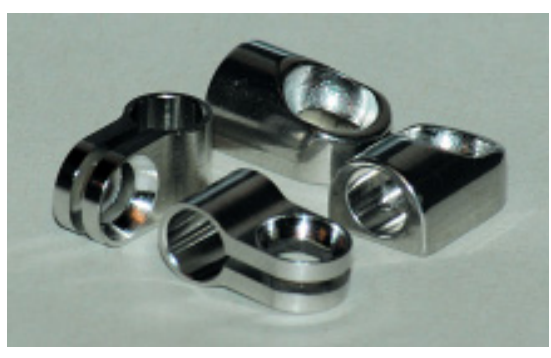

Abb.1 CRIF-Standard- und Endklammern. 


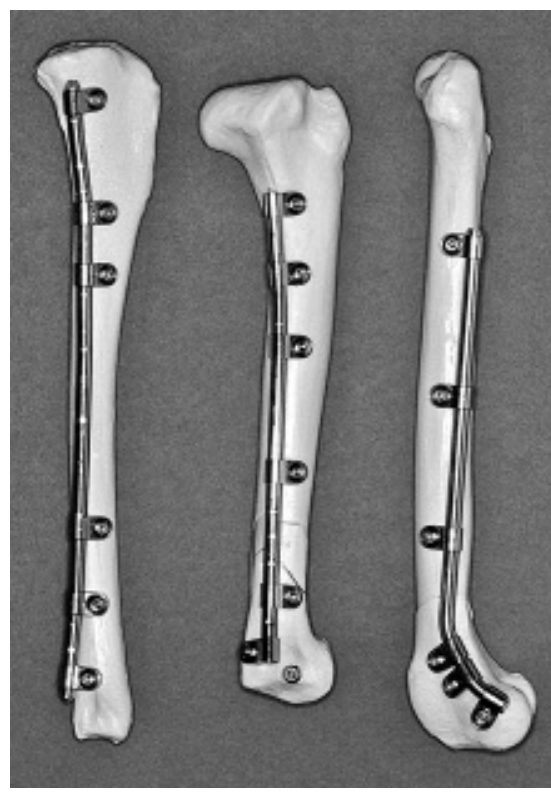

Abb. 2 Positionierung des CRIF an Tibia (medial), Humerus (medial) und Femur (lateral) (aus VCOT 3/2004 [23] mit freundlicher Genehmigung des Schattauer-Verlages).

mente zum Halten der Stäbe und Klammern entwickelt.

Handhabung und Eigenschaften des neuen Implantatsystems wurden zunächst in Ex-vivo-Studien zum Gebrauch des Implantates und der Instrumente [21] wie auch durch umfangreiche biomechanische Untersuchungen $[6,7,22]$ überprüft.

Diese zeigten, dass der CRIF hinsichtlich seiner Biege- und Torsionssteifigkeit mit AO-Standardplatten vergleichbar ist [22].

In der Klinik wurde das System bislang insbesondere bei Kleintieren an Wirbelsäule, Skapula, Becken und langen Röhrenknochen eingesetzt [23].

\section{Patienten mit Pseudarthrose}

An der Ludwig-Maximilians-Universität München kam das CRIF-System von Oktober 1999 bis Dezember 2003 bei elf Hunden und drei Katzen wegen einer atrophen $(\mathrm{n}=2)$, oligotrophen $(\mathrm{n}=1)$ bzw. hypertrophen $(n=11$, davon 8 infiziert) Pseudarthrose an Femur $(\mathrm{n}=7)$, Tibia $(n=4)$, Humerus $(n=2)$ und Radius $(\mathrm{n}=1)$ zum Einsatz. Die der Pseudarthrose vorangegangene Primärosteosynthese war mit Platte $(\mathrm{n}=8)$, Marknagel $(n=5)$ und Fixateur externe $(n=1)$ erfolgt. Das Körpergewicht der Patienten lag zwischen 5 und $53 \mathrm{~kg}$.
Bei Patienten mit nicht heilenden Unterarm- und Unterschenkelfrakturen nach externer Transfixation wurde zuerst der Fixateur in einer Kurznarkose entfernt und die Gliedmaße unter dem Schutz eines Hartschalenverbandes (Cellacast ${ }^{\circledR}$ ) bis zur Abheilung der Hautwunden immobilisiert.

\section{Debridement}

Der Zugang zum Frakturgebiet erfolgte standardmäßig unter bestmöglicher Schonung der Weichteile. Nach Entnahme einer tiefen Tupfer- oder Gewebeprobe zur bakteriologischen Untersuchung und Resistenzbestimmung wurde Restmaterial früherer Osteosynthesen sowie sequestrierter Knochen entfernt. Die Frakturenden wurden nach Ausräumen des Spaltraumes im Sinne einer Enbloc-Ostektomie [1] mit der oszillierenden Säge parallel zum Frakturspalt knochenmaterialsparend exzidiert oder wenigstens der Markraum mit PridieBohrungen geöffnet und - soweit erforderlich - prominentes Kallusgewebe im Verlauf des Knochenschaftes abgetragen bzw. geebnet. Die mit der Ostektomie nicht erfassten Knochenlakunen im Bereich der Markhöhle wurden kürretiert.

Nach Spongiosatransplantation aus der proximalen Humerusmetaphyse wurden die Knochenenden unter kortikaler Abstützung reponiert und temporär mit einem Bohrdraht fixiert. Bei Pseudarthrosen am Unterschenkel musste zur weitgehend spaltfreien Adaptation die Fibula gekürzt werden.

\section{Osteosynthese}

Die endgültige Frakturfixation erfolgte mit einem oder zwei CRIF und mindestens mit 2, überwiegend jedoch mit 3 Klammern an jedem Hauptfragment.

Dazu wurde zuerst der CRIF-Stab in benötigter Stärke und Länge dem Knochen anmodelliert, dann die erforderliche Anzahl Klammern aufgeschoben und der jeweiligen Fraktursituation entsprechend angeordnet. Eine temporäre Fixation des CRIF am Knochen erfolgte mit üblichen Plattenhalte- oder Zweipunktzangen. Aufgrund der geringen Überlagerung des Knochens durch das Implantat konnte die Reposition stets gut überprüft werden. Selbst bei einer Kombination von zwei CRIF war die Sicht auf das Frakturgebiet nahezu ungestört.
Eine alternierende Platzierung der Klammern ermöglichte die Winkelung der Schraubenachsen, um Knochendefekten auszuweichen und mehrere Schrauben auf engem Raum unterzubringen. Periphere Klammern wurden zuerst fixiert.

Achsenfehlstellungen konnten unter Durchleuchtungskontrolle in der Regel durch geringgradiges Lösen einer Schraube und Drehen des Stabes in der Klammer korrigiert werden.

Schließlich wurden nacheinander die restlichen Klammern befestigt. Das Anzugsdrehmoment wurde so gewählt, dass sich die Lippen der Klammern berührten.

Die Weichteildeckung des CRIF war an allen Lokalisation unproblematisch und erfolgte schichtweise. Wenn die tieferen Gewebeschichten nicht spannungsfrei über den Implantaten adaptierbar waren, wurden die Fasziennähte am Stab verankert. An Unterarm und Unterschenkel musste das Unterhautgewebe etwas mobilisiert werden, um das Implantat sicher zu decken.

\section{Nachsorge}

Die Patienten verblieben im Allgemeinen zwei Tage stationär und erhielten für eine Woche das nichtsteroidale Antiphlogistikum Carprofen $(1 \times$ tgl. $4,4 \mathrm{mg} / \mathrm{kg})$ zur postoperativen Analgesie. Antibiotika wurden strikt perioperativ $(25 \mathrm{mg} / \mathrm{kg} \mathrm{Ce}-$ phazolin i.v. 30 Minuten vor Operationsbeginn) und nur bei Infektionsverdacht über einen längeren Zeitraum oral appliziert. Bei infizierten Frakturen wurde die Antibiose erforderlichenfalls nach dem Antibiogramm umgestellt. Nach Unterarm- und Unterschenkelosteosynthesen wurde die Wunde bis zum Fädenziehen durch einen Polsterverband geschützt. Des Weiteren bestand die Nachsorge bei Hunden in Leinenzwang und bei Katzen in Wohnungshaltung. Die Dauer der Bewegungseinschränkung richtete sich nach dem Röntgenbefund. In der Regel wurde bis zur Konsolidierung der Fraktur in sechswöchigen Intervallen radiologisch kontrolliert. Bei konsolidierten Frakturen wurde den Tierbesitzern routinemäßig eine Implantatentfernung empfohlen.

\section{Heilungsverlauf und Ergebnisse}

Alle Patienten belasteten die zuvor geschonte Gliedmaße zunehmend und wie- 

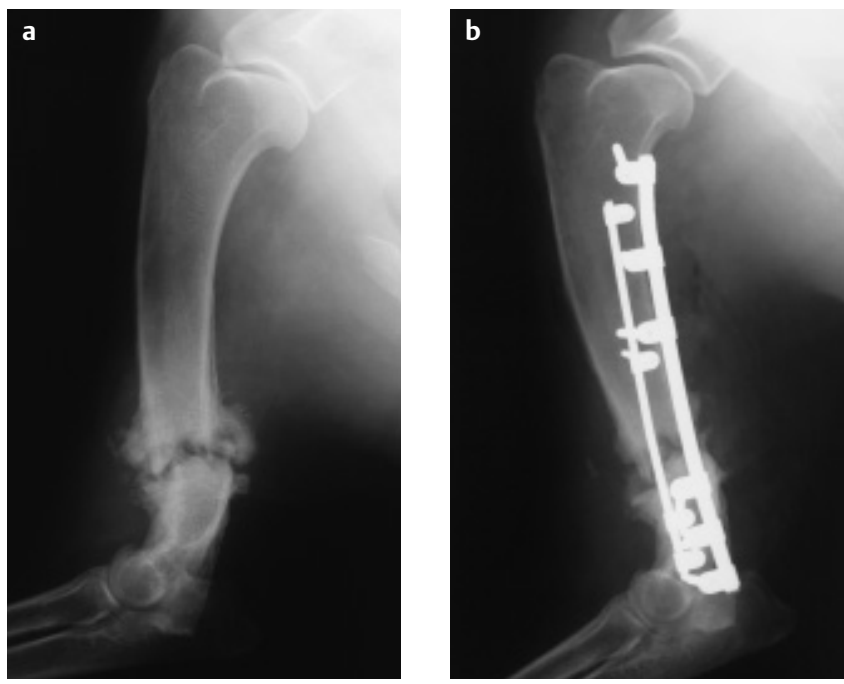

Abb. 3 Neufundländer, 5 Jahre, 50 kg KM: Hypertrophe Pseudarthrose des Humerus nach instabiler Marknagelung. Zustand nach Metallentfer-
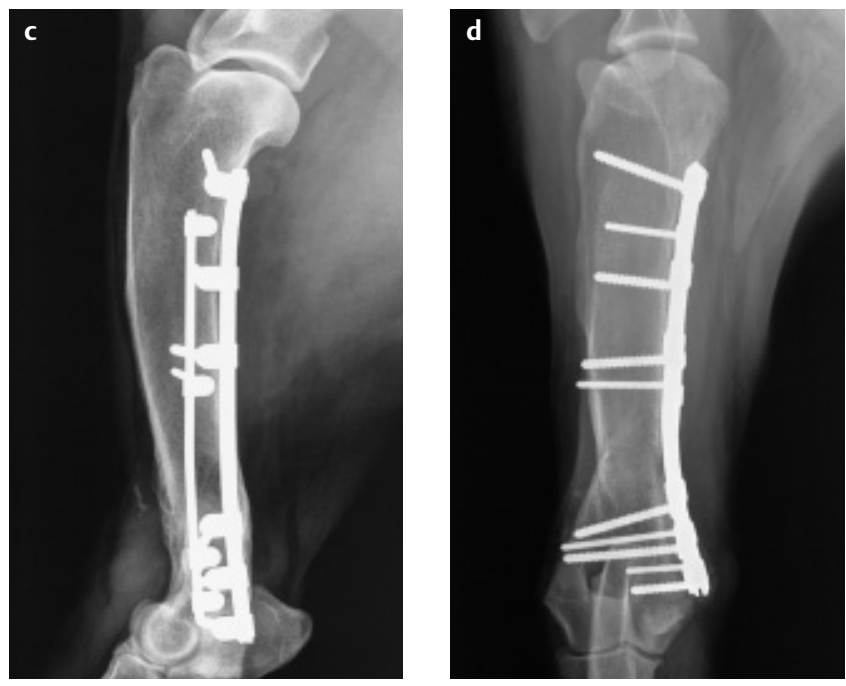

nung (a) und Reosteosynthese mit Doppel-CRIF der 2,7er und 3,5er Dimension (b). Situation nach 5 Monaten (c, d). sen einen progredienten Muskelzuwachs auf.

Eine vollständige Konsolidierung der Pseudarthrose wurde durchschnittlich nach 11 Wochen festgestellt.

Der Heilungsverlauf konnte röntgenologisch selbst bei einem Doppel-CRIF in beiden Projektionen gut verfolgt werden, da der Knochen nur gering vom Implantat überlagert wurde (Abb.3). Nur bei einem Patienten musste der CRIF aufgrund beginnender „stress protection“ nach 2 Monaten gegen einen schwäche- ren ausgetauscht werden (Abb.4). Weitere Komplikationen traten nicht auf. Obgleich das Implantat besonders am Trochanter major, im distalen Femurbereich und an der Tibia unter der Haut zu fühlen war, wurden äußerlich keine Weichteilirritationen beobachtet.

\section{Implantatentfernung}

Bei zehn Hunden und einer Katze wurde nach Konsolidierung der Fraktur eine Implantatentfernung durchgeführt. Anzeichen für eine persistierende Infektion bestanden nicht. Die Klammern waren häu- fig knöchern ummantelt und der Schlitz in der Klammer von Knochen ausgefüllt. Nach Entfernung der Schrauben konnten die Klammern mit einem Raspatorium mobilisiert werden. Der Stab lag reizlos in einem Gewebeschlauch und ließ sich leicht, oftmals mit den Klammern zusammen, hindurchziehen.

Dies ermöglichte bei proximal und distal platzierten Klammern einen minimalinvasiven Zugang über zwei kleine Inzisionen.
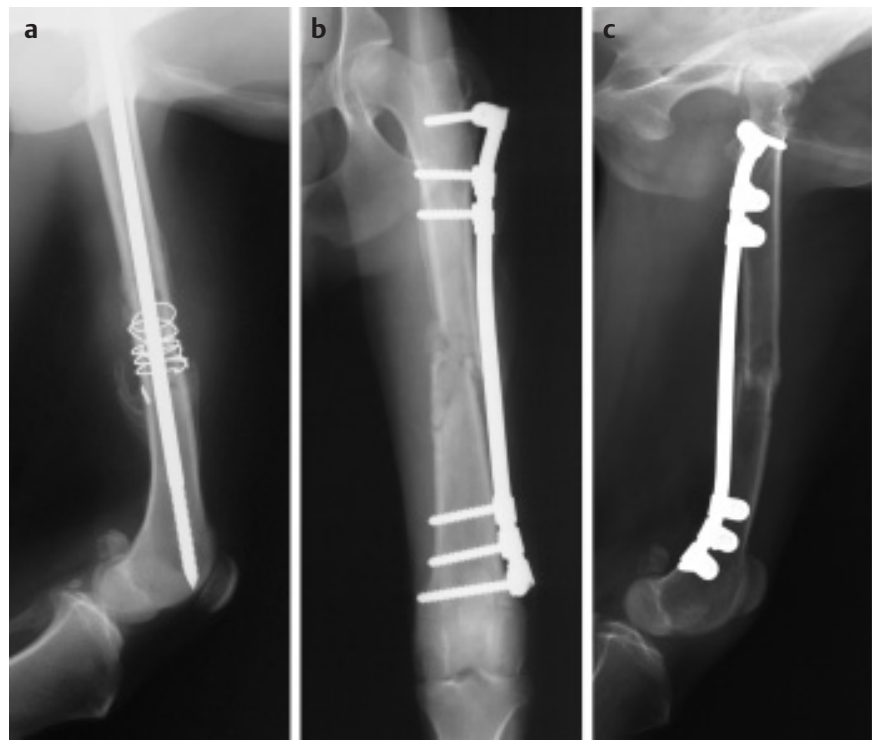

Abb. 4 Podenco Ibicenco, 1 Jahr, 15 kg KM: Oligotrophe Femurpseudarthrose 3 Monate nach instabiler Fixation mit Gewindenagel und Drahtcerclagen (a). Zustand nach Explantation, Anfrischen des Frakturspaltes, Spongiosatransplantation und Stabilisation mit 3,5er CRIF (b), sowie 2 Monate später mit lateraler Patellaluxation und beginnender
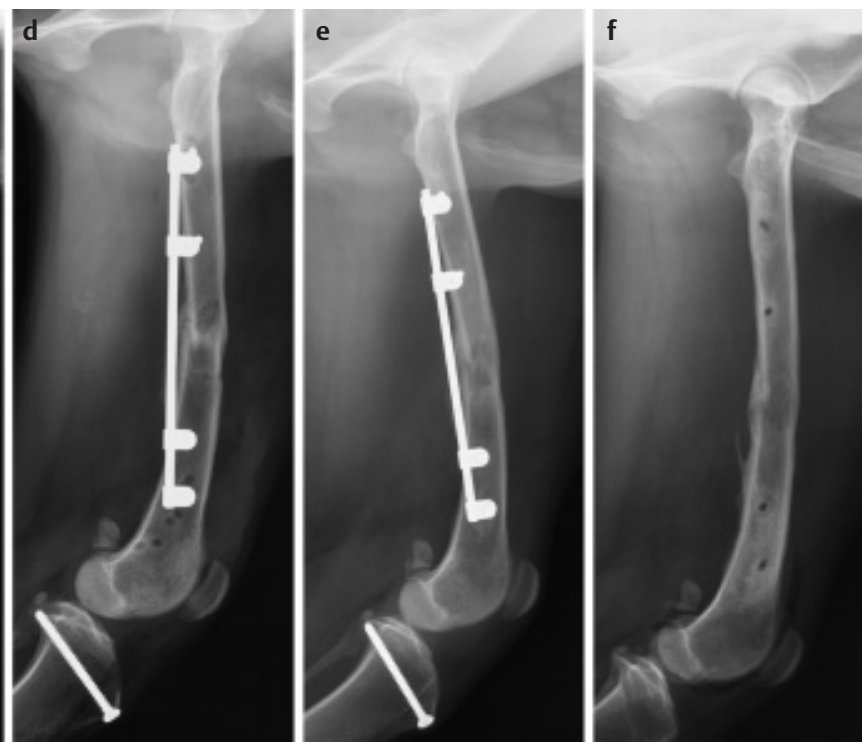

Stress Protection (c). Situation nach Transposition der Tuberositas tibiae nach medial und Dynamisierung der Fixation durch Austausch des 3,5er CRIF gegen 2,7er CRIF (d). 5 Monate nach Revision Femur remodelliert, Tuberositasosteotomie konsolidiert (e), Situation nach Implantatentfernung (f). 

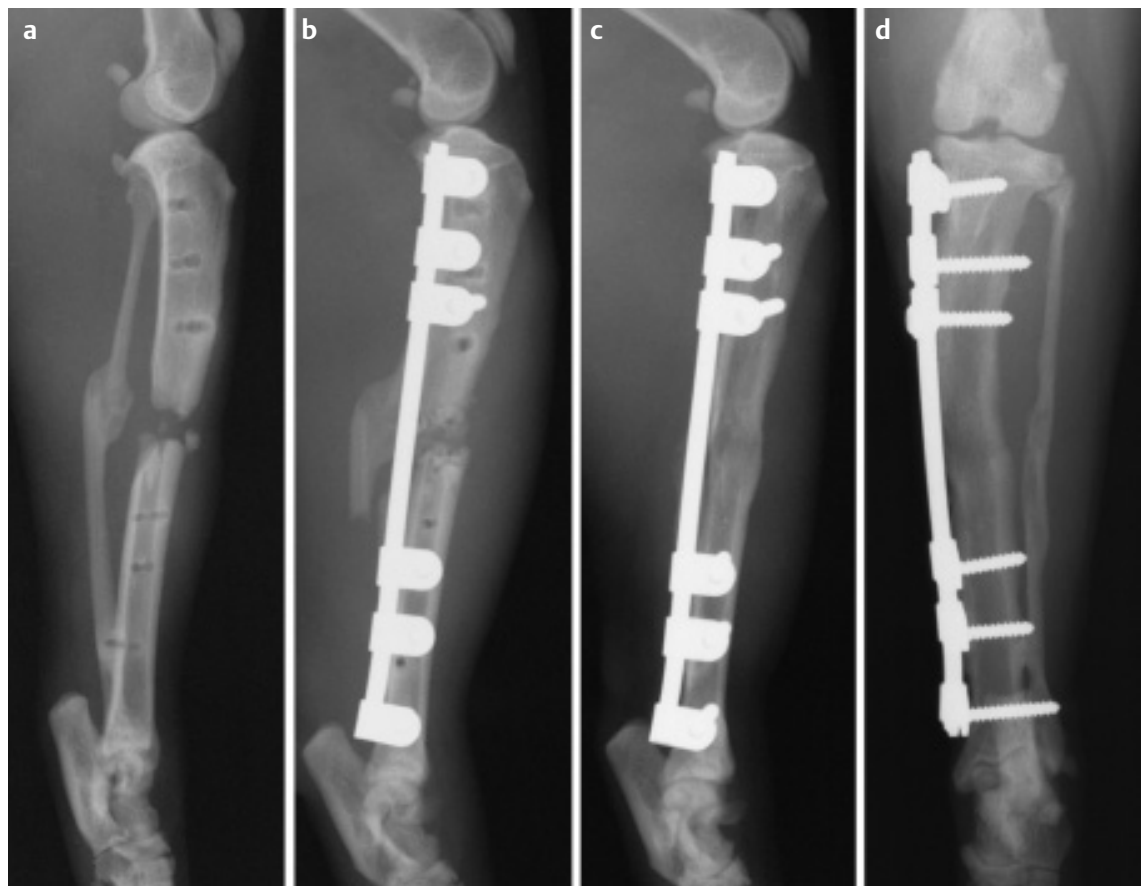

Abb. 5 Katze, 4 Jahre, 5 kg KM: Defektpseudarthrose der Tibia 10 Wochen nach Primärosteosynthese einer offenen Fraktur 3. Grades mit Typ-II-Fixateur externe (a). Fixation der Tibia mit 2,7er CRIF nach Kürzung der Fibula, Anfrischen der Frakturenden und Spongiosaeinlagerung (b). Komplikationslose Heilung 4 Monate nach Revision (c, d) (aus VCOT 3/2004 [23] mit freundlicher Genehmigung des Schattauer-Verlages).

\section{Diskussion}

Im Gegensatz zum Menschen ist beim Tier eine übungsstabile Osteosynthese nicht ausreichend.

Vielmehr erfordern die hier limitierten Möglichkeiten einer konsequenten Ruhighaltung den Einsatz belastungsstabiler Verfahren, die bis zur Konsolidierung der Fraktur einen sicheren Halt bieten, ohne die Remodellierung des Knochens zu behindern. Bei der Wahl der Implantatdimension muss neben der Körpergröße, der Lokalisation und Art der Fraktur auch das Temperament des Tieres eine Berücksichtigung finden. So kann es infolge Fehleinschätzung selbst bei Osteosynthesen erfahrener Operateure durch zu schwache oder zu rigide Fixation zu Komplikationen kommen. Die Pseudarthrose ist dennoch ein vergleichsweise seltenes Ereignis, das bei regelrechter Behandlung keine ungünstige Prognose hat.
Während nicht infizierte Pseudarthrosen beim Kleintier überwiegend durch Debridement und Plattenosteosynthese versorgt werden $[1,10]$, sind bei infizierten Pseudarthrosen transkutane Fixationen mit äußerer Schienung - unter anderem auch der Ringfixateur [15] - vorzuziehen, da Implantate mit engem Kontakt zum infizierten Frakturbereich eine persistierende Osteomyelitis begünstigen [14, 16]. Die für das Tier vorzuziehende Fixationsmethode stellt jedoch die interne Fixation dar, minimiert sie dank wegfallender Verbandwechsel die Häufigkeit der Tierarztbesuche bei zugleich hohem Tragekomfort.

Wir konnten mit dem Clamp Rod Internal Fixator (CRIF) bei allen von uns so versorgten Kleintierpatienten mit Pseudarthrose innerhalb kurzer Zeit eine Konsolidierung erreichen (Abb.5 und $\mathbf{6}$ ).

Neben ausreichender Stabilisierung dürfte sich der geringe Knochenkontakt dieses Fixationssystems heilungsfördernd ausgewirkt haben.

Mit seiner reduzierten Knochenauflage verfolgt der CRIF ebenso wie das Zespol-Prinzip [17], der „PC-Fix“ [20], die „No Contact Platte“ [3] und die „Locking Compression Plate“ [5] die Philosophie einer Schonung des periostalen Blutflusses bei zugleich stabiler Fixation im Sinne der biologischen Osteosynthese [12].

Der CRIF vereint die Vorteile der externen und internen Fixation gleichsam in einem „Fixateur interne“. Als vereinfachte Version ähnlicher Systeme aus der Wirbelsäulenchirurgie beim Menschen [18] bietet er ein hohes Maß an Vielseitigkeit, wie sie in der Tiermedizin aufgrund der
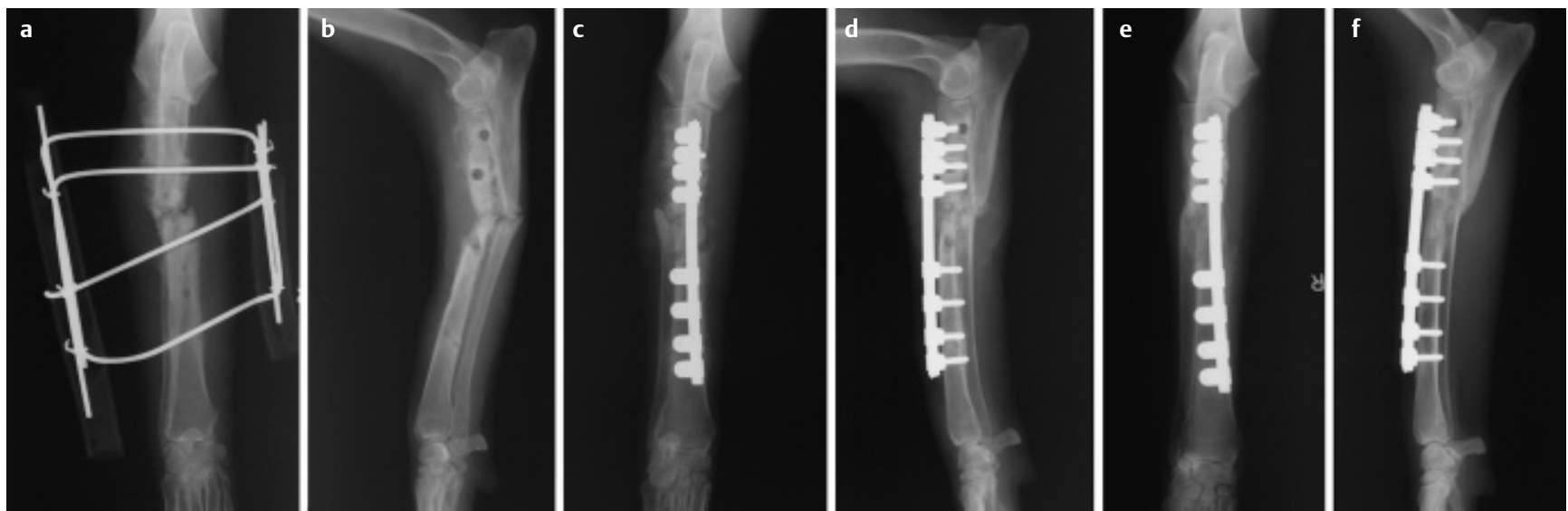

Abb. 6 Kleiner Münsterländer, 7 Jahre, 22 kg KM: Infizierte Radius- und Ulnapseudarthrose 4 Monate nach perkutaner Transfixation mit bilate-

teurs (b) Reosteosynthese des Radius mit 3,5er CRIF (c, d). Situation 5 Monate post revisionem (e, f). 
anatomischen Vielfalt im Knochenbau und der Größenunterschiede besonders erwünscht ist. So bietet der CRIF eine Längenvariabilität, die sonst nur die abschneidbare Veterinärplatte (VCP) und die Rekonstruktionsplatte (RCP) aufweisen [16]. Aufgrund seiner höheren Steifigkeit [22] erlaubt er im Gegensatz zu diesen aber auch den Einsatz bei Hunden mit großem Körpergewicht.

Besonders vorteilhaft ist die beliebige Anordnung der Klammern zu beiden Seiten des Stabes, womit das Implantat der jeweiligen Knochenoberfläche und Fraktursituation angepasst werden kann. Zudem können durch enge Aneinanderreihung der Klammern mehr Schrauben auf kurzer Strecke angebracht werden als bei herkömmlichen Platten. Damit vereinfacht der CRIF die Platzierung von mindestens zwei Schrauben selbst in einem kurzen Hauptfragment. Ein weiterer Vorteil ist die Korrigierbarkeit der axialen Ausrichtung während der Operation durch Drehen des Stabes in einer nicht vollständig fixierten Klammer, ohne Schrauben umsetzen zu müssen.

Zur Erhöhung der Stabilität von diaphysären Frakturversorgungen wurde für Hunde bis $12 \mathrm{~kg}$ die Kombination von zwei schneidbaren Platten beschrieben. Da sie in „Sandwich“-Technik übereinander angebracht werden, führt diese Kombination nicht zu einer zusätzlichen Beeinträchtigung der periostalen Blutversorgung [2], wie sie bei der Verwendung von Platten generell möglich ist [9]. Bei schweren Hunden kann mit gleichem Ziel die Plattenosteosynthese mit einem intramedullären Kraftträger kombiniert werden, wobei ein zu dicker Marknagel die Schraubenplatzierung stören und darüber hinaus auch die endostale Durchblutung beeinträchtigen kann [8].

Dagegen erlaubt der CRIF eine parallele Anordnung gleicher oder verschiedener CRIF-Größen ohne Interferenz der Schrauben. So ist eine den Dimensionen des Tieres angepasste Variation der Fixationssteifigkeit möglich, ohne die Knochendurchblutung nennenswert zu stören.

Selbst bei Kombination von zwei CRIF besteht im Vergleich zur Platte eine sehr geringe Knochenabdeckung, was intraope- rativ die Kontrolle der Reposition erleichtert und postoperativ die Röntgeninterpretation der Frakturheilung vereinfacht. Im Falle einer „stress protection“ bietet sich bei der CRIF-Kombination eine Dynamisierung des Knochens durch Teilimplantatentfernung an. Diese kann, bei geeigneter Anordnung der Klammern jeweils an den Stabenden, über kurze Inzisionen erfolgen und erlaubt dem Patienten eine rasche Wiederherstellung der Mobilität.

Zusammenfassend darf man feststellen, dass der CRIF alle Anforderungen an ein tiermedizinisches Allround-Implantat erfüllt: Variabel in der Länge, anpassungsfähig in der Steifigkeit, durch engen Schraubenabstand auch kurzen Fragmenten Halt bietend, mit Standardschrauben verschiedener Größe verwendbar und wirtschaftlich sowohl in der Lagerhaltung als auch im Gebrauch.

\section{Literatur}

${ }^{1}$ Blaeser LL, Gallagher JG, Boudrieau RJ. Treatment of biologically inactive nonunions by a limited en bloc ostectomy and compression plate fixation: a review of 17 cases. Vet Surg 2003; 32: $91-100$

2 Bruese S, Prieur WD. Anwendung der Veterinärabschneideplatte (VCP) in 160 Fällen. Tieraerztl Prax 1996; 24: 581 -9

${ }^{3}$ Brunnberg L, Horst C, Gagel A et al. Die No Contact Plate (NCP) Osteosyntheseplatte Ein neues biologisches Implantatsystem. Kleintierpraxis 1998; 43: 579-91

${ }^{4}$ Duhautois B. Use of interlocking nails for diaphyseal fractures in dogs and cats: 121 cases. Vet Surg 2003; 32: 8-20

${ }^{5}$ Frigg R. Locking compression plate (LCP). An osteosynthesis plate based on the dynamic compression plate and the point contact fixator (PC-Fix). Injury 2001; 32: 63-6

${ }^{6}$ Haerdi C, Dalla Costa R, Auer JA et al. Mechanical comparison of 3 different clamp and 2 different rod types of a new veterinary internal fixation system, 4.5/5.5-mm CRIF. Vet Surg 2003; 32: $431-8$

${ }^{7}$ Haerdi-Landerer C, Steiner A, Linke B et al. Comparison of double dynamic compression plating versus two configurations of an internal veterinary fixation device: results of in vitro mechanical testing using a bone substitute. Vet Surg 2002; 31: $582-8$

${ }^{8}$ Hulse D, Hyman W, Nori M et al. Reduction in plate strain by addition of an intramedullary pin. Vet Surg 1997; 26: $451-9$

${ }^{9}$ Jain R, Podworny N, Hupel TM et al. Influence of plate design on cortical bone perfusion and fracture healing in canine segmental tibial fractures. J Orthop Trauma 1999; 13: $178-86$

${ }^{10}$ Knobloch S. Komplikationen nach Osteosynthese von Schaftfrakturen langer Röhrenknochen bei Hund und Katze. Ursachen,
Therapie und Ergebnisse in den Jahren 1980-1987. Diss med vet LMU München 1990

${ }^{11}$ Kraus KH, Kadiyala S, Wotton $\mathrm{H}$ et al. Critically sized osteo-periosteal femoral defects: a dog model. J Invest Surg 1999; 12: 115-24

${ }^{12}$ Matis U. Biologische Osteosynthese. In: Bonath $\mathrm{KH}$, Prieur WD. Kleintierkrankheiten Band 3: Orthopädische Chirurgie und Traumatologie. Stuttgart: Ulmer 1998: 230-8

${ }^{13}$ Matis U, Köstlin RG, Brunnberg L. Fehler bei der Frakturbehandlung beim Kleintier und deren Folgen. Berl Münch Tierärztl Wschr 1985; 98: 173 - 80

${ }^{14}$ Millis DL, Jackson AM. Delayed unions, nonunions, and malunions. In: Slatter D. Textbook of small animal surgery. Philadelphia; Saunders 2003: 1849-61

${ }^{15}$ Owen MA. Use of the Ilizarov method to manage a septic tibial fracture nonunion with a large cortical defect. J Small Anim Pract 2000; 41: $124-7$

${ }^{16}$ Piermattei DL, Flo GL. Brinker, Piermattei, and Flo's Handbook of small animal orthopedics and fracture repair. Philadelphia: WB Saunders 1997; 154-62

${ }^{17}$ Ramotowski W, Granowski R. Zespol - An original method of stable osteosynthesis. Clin Orthoped Rel Res 1991; 272: 67-75

${ }^{18}$ Richter M, Wilke HJ, Kluger $P$ et al. Biomechanical evaluation of a new modular rodscrew implant system for posterior instrumentation of the occipito-cervical spine: in-vitro comparison with two established implant systems. Eur Spine J 2000; 9: $417-25$

${ }^{19}$ Robins GM. Delayed union and nonunion. In: Brinker WO, Olmstead ML, SumnerSmith G, Prieur WD. Manual of internal fixation in small animals. Berlin: Springer, 1998: $227-40$

${ }^{20}$ Savoldelli D, Montavon PM. Clinical handling: small animals. In: Point Contact Fixator: Part 1. Injury 1995; 26: 47-50

${ }^{21}$ Schwandt CS. User manual for the 4.5/5.5 mm CRIF: Developement of a new veterinary internal fixation system, from the prototype to the final version. Thesis University of Bern, Switzerland 2001

${ }^{22}$ Zahn K, Matis U et al. Biomechanical comparison of the CRIF system and commonly used AO bone plates. ESVOT-VOS $1^{\text {st }}$ World Orthopaedic Veterinary Congress, Munich 2002

${ }^{23}$ Zahn K, Matis U. The Clamp Rod Internal Fixator - Application and results in 120 smal animal fracture patients. Vet Comp Orthopaed Traumatol 2004; 3: im Druck

\section{Dr. med. vet. Klaus Zahn \\ Assistenzarzt \\ Prof. Dr. med. vet. Ulrike Matis \\ Klinikvorstand}

Chirurgische Tierklinik

der LMU-München

Veterinärstr. 13

D-80539 München 